\title{
Evaluation of the supply capacity of livelihood service facilities in the post-epidemic era
}

\author{
Ruipeng Zhang ${ }^{1, *}$, Changquan $\mathrm{Su}^{1}$, and Junwei Huo ${ }^{1}$ \\ ${ }^{1}$ College of Architecture and Art, Hefei University of Technology, Hefei, China
}

\begin{abstract}
Livelihood supply security is a critical issue when a public health emergency comes, and the layout planning of existing commercial facilities in China is not yet able to meet the livelihood supply in emergency situations. Evaluating the layout plan of existing commercial facilities is the first step to optimize them. Based on the theory of community "living circle", this study takes the central urban area of Hefei City as the scope of research, uses the big data platform and GIS to analyze the accessibility of commercial facilities for people's livelihood supply, conducts comprehensive evaluation at the community level, and explores the development direction of optimizing the planning and layout of commercial facilities for people's livelihood supply in the community living circle. The final analysis concludes that the layout planning of livelihood supply commercial facilities in areas with relatively low degree of construction at the edge of the central city of Hefei is unable to meet the basic living needs of residents under normal conditions. The problem is less obvious in the core area of the central city, but is further magnified in the face of sudden public health events, and its problematic area is more obviously expanded.
\end{abstract}

\section{Introduction}

The sudden new crown pneumonia epidemic swept around the world at the end of 2019, and the socioeconomic development and daily life of the people suffered an unprecedented impact. Nowadays, the epidemic has been better prevented and prevented in China, but urban construction still reveals many problems, such as insufficient livelihood supply capacity. This indicates that urban development has some shortcomings in grassroots livelihood and medical care. In this context, the construction of the city should focus on promoting the construction and improvement of the livelihood supply commercial facility system, and should consider the two states of sudden epidemic and epidemic normalization. In addition, the relationship between facilities and the daily needs of community residents should be considered, and the community life supply of the people should be effectively guaranteed through the optimization of spatial layout to improve the resilience of the city.

This paper takes the construction of livelihood supply service facilities in the context of the future epidemic as the starting point, introduces the community "living circle" theory, and takes the configuration requirements of different levels of "living circles" for the construction of livelihood supply commercial facilities as the basis to analyze and study the rational layout of livelihood supply facilities under the epidemic prevention and control. The study will analyze and research how to reasonably layout the facilities under the epidemic prevention and control. The aim is to explore the development direction of optimizing the planning and layout of commercial facilities for livelihood supply in community living circles [1].

\section{Research Concepts and Methods}

\subsection{Research Concepts}

\subsubsection{Living Circle Residential Area}

After the introduction of the latest standard, the old standard is divided into "residential area, district and group" according to the number of households or population size, and the new standard is divided into "15minute living circle residential area, 10-minute living circle residential area, and five-minute living circle residential area" based on the principle that residents can meet their basic living needs within a reasonable walking distance. residential areas, five-minute living areas, residential neighborhoods" four levels. The concept of "living circle" aims to provide a fair environment for residents to enjoy quality space, and is a reflection of the people-oriented approach, emphasizing the change from "service radius" to "time radius".

\subsubsection{Facilities for people's livelihood services}

The definition of commercial facilities for people's livelihood supply should include commercial facilities that meet residents' needs in all aspects of life, including clothing, food, housing and transportation. When a

\footnotetext{
* Corresponding author: zhangrphfut@163.com
} 
public health emergency comes, the livelihood needs of residents are somewhat restricted and transformed into a necessary basic need. According to the relevant policy documents issued by the state, citing its definition of essential supplies for people's livelihood during an epidemic: epidemic prevention supplies such as masks, antiviral drugs, disinfection and sterilization supplies, relevant medical devices, and basic livelihood commodities such as food, oil, meat, eggs, vegetables and milk related to the daily life of the masses. The corresponding commercial facilities for the supply of people's livelyhood are also transformed into convenience stores and pharmacies accordingly [2]. This is consistent with the definition of "five-minute living circle" in the Urban Residential Area Planning and Design Standards (GB 50180-2018), which states that "the residential area is divided by the principle that residents can meet their basic living needs within five minutes' walk. " which is consistent with the living needs expressed in the "Fiveminute Living Area".

\subsection{Evaluation methods}

Accessibility refers to how easy it is to reach a place from another place. In this paper, we use quantitative analysis method, based on the analysis of the walkable accessibility of commercial facilities for people's livelyhoods, and combine road traffic information to classify "5-minute service area", "10-minute service area", and "15-minute service area". 15-minute service area", and analyze and evaluate the residential areas. This analysis method is a more scientific and reasonable simulation, which breaks the method of accessibility analysis by distance measurement in conventional research, and follows the idea of "service radius" to "time radius" in the new version of the specification [3].

\section{Study area and data}

\subsection{Study area}

Hefei, the central urban area, the regional scope of this study is the central urban area of Hefei city, the object is all kinds of livelihood supply commercial facilities and residential points.

\subsection{Research Data}

The data used in this study include three parts, the traffic road data of Hefei city, the distribution point data of livelihood supply commercial facilities within the central city of Hefei city, and the distribution point data of residential areas within the central city of Hefei city. The road data comes from the OpenStreetmap database, which is an open geographic database with geographic data provided by users spontaneously, and the data provided by this database has good quality and accuracy has been verified. The supply facility point and residential area layout data come from the POI data crawled by OpenStreetmap (Figure 2), which is often used in big data analysis.

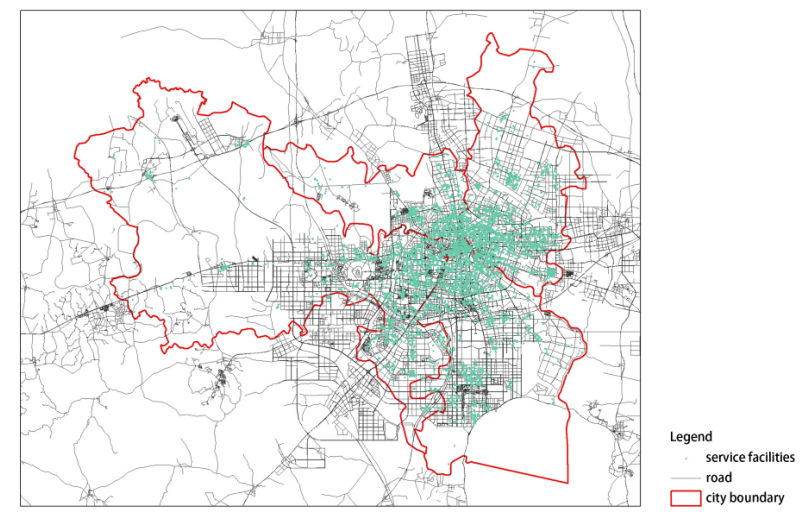

Fig. 1. Spatial distribution of POI of commercial facilities in the central city of Hefei.

\subsubsection{Facility point data and its processing}

In this study, the functional requirements of livelihood supply commercial facilities are used to screen the Gode POI data, and the finalized classifications of POI data in this study are: shopping malls, convenience stores, supermarkets, and general markets under shopping service facilities, and medical and healthcare sales stores under healthcare services [4].

This study will briefly simulate the state of livelihood supply commercial facilities under the influence of the epidemic, and by crawling the 2019 Gao De Map POI data and 2020 Gao De Map POI data for comparison, it is found that the total number of POI data points in 2020 has decreased and is concentrated in the categories of shopping malls and supermarkets, and the analysis concludes that the reason for the decrease in the total number of data points is due to the public health emergencies in 2020 coming, the larger-scale facility points will be affected by the epidemic prevention and control policy and stop supplying, and the smaller-scale facility points will easily continue to maintain their functions under the epidemic due to their flexibility and resilience. Therefore, the 2019 GOD POI data was selected as the data source before the arrival of a public health emergency, and the 2020 GOD POI data was selected as the data source before the arrival of a public health emergency.

\subsubsection{Basic traffic data and its processing}

The crawled road data is simplified and processed to remove some redundant road information, and the final retained roads are main roads, secondary roads, feeder roads and walking roads. After the road data is supplemented with fields, the length of the road is geometrically calculated, the walking speed of a person is assigned $(4 \mathrm{~km} / \mathrm{h}$ as the standard $)$, and the walking time is calculated from the first two. After the information is added, the road data is checked and a network topology analysis of the road data within the study area is created to correct and correct any errors that occur.

After the processing of the basic data, a network data 
set is constructed for the road data for simulation. Since the element of this study is human walking activity, only a simple simulation of a single line is performed. By building the traffic network dataset, attributes such as intersection turns, connectivity, and distance cost are set, where the cost of passage is selected as walking time. The above completes the initial construction of the traffic network [5].

\subsubsection{Resident point data and its processing}

Based on the range of facility service areas derived from the data network analysis method, the livelihood supply of residents under the impact of the epidemic was further studied by combining the data of urban residents' distribution points [6]. First, the coverage area of each livelihood supply facility service area is counted, and then the coverage area of livelihood supply commercial facility service area under the normal situation is compared with that under the epidemic situation to analyze the impact of the epidemic on livelihood supply. Second, according to the coverage area of the facility service area, the number of settlements located in each level of service area was counted, and the impact of livelihood supply by the epidemic on livelihood supply was analyzed through the change in the number of settlements within the service area.

\section{Evaluation of the supply capacity of people's service facilities}

\subsection{People's livelihood supply commercial faci- lities service area evaluation}

According to the distribution of service areas at all levels of livelihood supply commercial facilities under normal conditions (Figure 2), it can be seen that most of the areas in the central city of Hefei are within the 5-minute service range, a small part of the scattered areas are in the 10-minute service range, and a large area extending outward from the central city is also in this service range. A large part of the area is in the 5-minute service range, and the service area of livelihood supply facilities can cover better than the border outward, there are some areas with 5-minute service area, but most of them are not able to reach this standard, or even far beyond this standard.

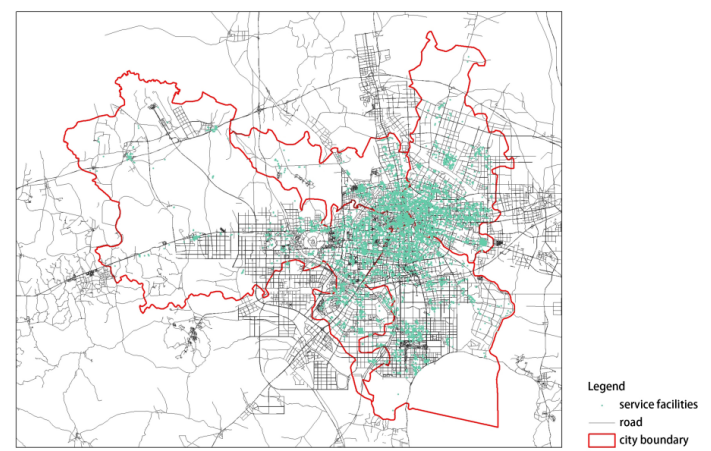

Fig. 2. Distribution of livelihood supply commercial facilities service area under normal conditions.
According to the simulated distribution map of service areas of livelihood supply facilities at all levels under the epidemic (Figure 3) compared with the distribution map of service areas of livelihood supply commercial facilities under the norm, it can be seen that the 5-minute service area in the central city of Hefei under the epidemic shifts toward Yaohai District, and the 10-minute service area starts to appear in the central city to a greater extent, and the 15-minute service area spreads within the boundary of Hefei Beltway - North Second Ring Road, and more areas are located beyond the 15-minute service area.

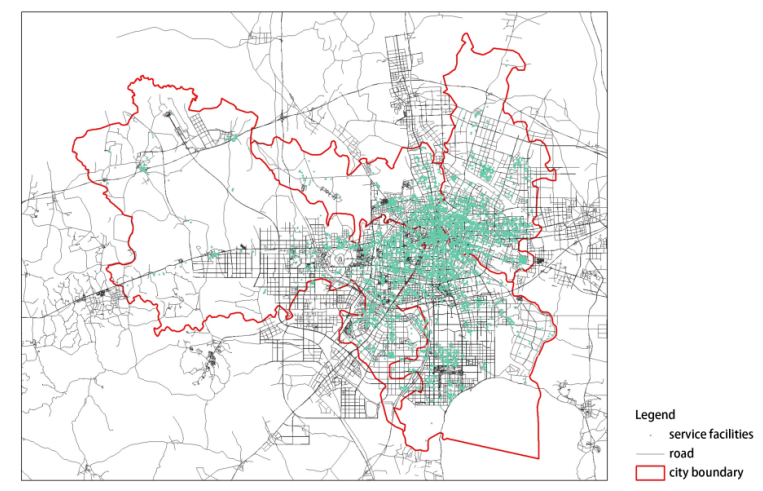

Fig. 3. Distribution of service areas of livelihood supply facilities under the epidemic.

By observing the layout of service areas of commercial facilities for people's livelihood before and after the epidemic, we can conclude that:

- Before the epidemic, the number of commercial facili-ties for people's livelihood outside the area of Hefei Bypass-North Second Ring Road was obviously insuffi-cient.

- The epidemic had a negative impact on the service area of commercial facilities for people's livelihood, and the impact was more obvious for the areas on the edge of Hefei Bypass-North Second Ring Road. The impact is more obvious in the marginal areas of the Hefei Bypass North Second Ring Road, mainly in the reduction of the 5-minute service area, the extension of the 10-minute and 15-minute service areas to the city center and the emergence of more areas not covered by the 15 -minute service area.

\subsection{Evaluation of residential points within each class of service area}

Considering that residents would reduce their travel time under the influence of the epidemic, the feasibility was classified as comfortable by combining the circle of life theory, with 5 minutes reachable being comfortable, more than 10 minutes to 15 minutes reachable being classified as uncomfortable, and areas more than 15 minutes reachable were not considered in this study. Preepidemic data were also classified using the same criteria. To make the analysis results more intuitive, further quantitative analysis was performed based on the service area accessibility analysis chart of livelihood supply facilities, and the area covered by each comfort zone, the 
percentage of area covered by service areas of different comfort levels before and after the epidemic, and the number of residential points covered were derived.

Table 1. The area of the service area of the livelihood supply facilities at all levels during non-epidemic situations.

\begin{tabular}{|c|c|c|}
\hline Service Area Level & $\begin{array}{c}\text { Area of each } \\
\left.\text { grade } \mathbf{( k m}^{2}\right)\end{array}$ & $\begin{array}{c}\text { Cumulative } \\
\text { area } \mathbf{( k m}^{2} \text { ) }\end{array}$ \\
\hline 5 minutes service area & 118.974 & 118.974 \\
\hline 10 minutes service area & 113.256 & 232.232 \\
\hline 15 minutes service area & 138.748 & 370.980 \\
\hline
\end{tabular}

Table 2. Area at all levels of the service area of the livelihood supply facilities at the time of the epidemic.

\begin{tabular}{|c|c|c|}
\hline Service Area Level & $\begin{array}{c}\text { Area of each } \\
\left.\text { grade } \mathbf{( k m}^{\mathbf{2}}\right)\end{array}$ & $\begin{array}{c}\text { Cumulative } \\
\text { area } \mathbf{( k m}^{2} \mathbf{)}\end{array}$ \\
\hline 5 minutes service area & 94.764 & 94.764 \\
\hline 10 minutes service area & 96.036 & 190.802 \\
\hline 15 minutes service area & 118.923 & 309.725 \\
\hline
\end{tabular}

Analysis of Table 1 and Table 2 shows that:1) The total area of the service area of livelihood supply facilities reachable within 15 minutes before and after the impact of the public health emergency decreas-ed from $370.98 \mathrm{~km}^{2}$ to $309.725 \mathrm{~km}^{2}$ for all levels of service areas. (2)Before the arrival of the sudden public health event, the area of the service area to meet basic living needs (5-minute service area) in the central city of Hefei was $118.974 \mathrm{~km}^{2}$, and the area outside this service area was $252.006 \mathrm{~km}^{2}$, and the residential points located in the service area to meet basic living needs also accounted for only $59.82 \%$. (3) When the sudden public health event came, the residential points in the central city of Hefei The total number of settlements within the 5-minute service area was 3480, which was nearly 600 fewer than before the epidemic.

\subsection{Analysis of study results}

The following results can be derived from this study:

(1) The supply capacity of the urban fringe areas is obviously insufficient. The areas at the edge of the central city of Hefei with a relatively low degree of construction have the problem that the layout planning of their livelihood supply commercial facilities cannot meet the basic living needs of residents under normal conditions. The problem is less obvious in the core area of the central city, but is further magnified in the face of sudden public health events, and the area where the problem exists has expanded more obviously.

(2) The service time to meet the basic living needs is relatively long. The current service area to meet basic living needs in the central city of Hefei (5-minute service area area) accounts for only $59.82 \%$.

(3) The livelihood supply system is overly dependent on large commercial facilities. At present, large commercial facilities account for a high proportion of the livelihood supply commercial facilities in the central city of Hefei, causing the problem of over-reliance on large commercial facilities for the normal operation of the supply system. When an unexpected public health event comes, the supply system experiences a sudden drop in supply capacity when large commercial areas are suspended from business due to the restrictions of epidemic prevention and control requirements.

\section{Conclusion}

Livelihood supply is a guarantee for the basic life of residents, and this guarantee becomes more necessary in the face of sudden public health events. This paper concludes from research and analysis that there are certain drawbacks in the current layout planning of livelihood supply commercial facilities in the central city of Hefei, which cannot fully cope with the impact of sudden public health events. In order to strengthen urban resilience and enhance the city's ability to cope with sudden public health events, the livelihood supply commercial facilities in the problem areas should first be supplemented in terms of quantity, and secondly, the linkage development with online shopping mode can be sought by relying on urban convenience stores, which are small-scale livelihood commercial supply points with long business hours. How to establish a livelihood supply system for resilient cities is the core issue of optimizing the layout of livelihood supply commercial facilities, and will be a hot direction for future research in this field.

\section{Acknowledgments}

This work was supported by the National Student Innovation and Entrepreneurship Project - Research on the Livelihood Supply System of Resilient Cities (Reflections on the Difficulty of Community Grocery Shopping during the Epidemic) (202010359057).

\section{References}

1. Kong D, Wang H. A study on the layout planning of urban livelihood commercial supply in response to sudden infectious epidemic[J]. Planner, 2020, 36 (05): 109-112.

2. Zhou W. Study on the correlation between urban built environment and high-risk communities under the epidemic--based on a sample of seven urban communities in Hankou[J]. New Architecture, 2020 (06): 52-55.

3. Wang Z, Fei Y. Discussion on the evaluation method of community public service level from the perspective of "living circle"[J]. Intelligent Building and Smart City,2021(03):17-21.

4. Wei W, Hong M, Zhou J, Xia J. The evaluation method of urban basic public service facilities from the perspective of "urban people": the case of Wuhan City[J]. Urban Planning, 2020, 44(10):71-80.

5. Chen B, Li Y. The dilemma and innovation of urban livelihood public goods supply--reflection based on 
urban agglomeration[J]. Social Policy Research, 2021(01):39-52.

6. Yu Y. From traditional residential planning to community living circle planning $[\mathrm{J}]$. Urban Planning, 2019, 43(05): 17-22. 